\title{
Mean Velocity in the Asymmetric near Wake Behind a GAW(2) Airfoil
}

\author{
N. Subaschandar \\ Department of Mathematics and Computational Sciences, BIUST, Palapye, Botswana
}

\begin{abstract}
The two-dimensional asymmetric near wake behind a GAW(2) airfoil section is studied at low speeds. Mean velocity measurements have been made in the near wake. Mean velocity profiles in the near wake are presented. Also integral parameters based on the mean velocity profiles have been presented here. In the near wake region mean velocity profiles are seen to exhibit symmetry within a distance of about 60 trailing edge momentum thicknesses downstream of the trailing edge.
\end{abstract}

\section{Introduction}

Turbulent wake region is the region of flow behind the trailing edge of a body where the upstream boundary layers merge into one single shear layer. The evaluation and understanding of flow in the in the near wake is important as the flow in this region is still influenced by the upstream trailing edge conditions. Most of the investigations in the near-wake, theoretical as well as experimental are confined to the two-dimensional wake flows [1-4]. At the edge of the trailing edge of a streamlined body, where the boundary layers transform into a wake, the skin friction suddenly drops to zero, and at the same time, the wall restriction on the normal-towall velocity component is removed. In symmetric wakes, this allows mixing across the wake centreline and initiates inner near wake[1]. In asymmetric wakes, both mean flow and turbulent transport across the wake centreline takes place. Therefore, the relative intensities and the relative size of the turbulence on both sides become important in the development of wake. In addition, there is longer-range influence due to equalisation of the pressure field, when there is a pressure variation in the trailing edge region, as in the case of airfoil experiencing lift. In general, these effects appear as combined and interact with each other. Leuchter[5] studied asymmetric generated downstream of a flat plate by contouring the ceiling and the floor of the tunnel differently. The asymmetry obtained in this way is somewhat closer to the airfoil wake, but is mild and makes the external flow itself asymmetric. Studies[6,7], on the other hand, used a resistance such as a screen to retard flow on one side of the body. The asymmetric wake generated in this way is more akin to a mixing layer between two different streams of different total pressures, and they show wake-like behaviour only in the region close to the trailing edge, and quickly start to show the mixing layer type of profiles. The aim of the present study is to analyse and understand near wake flow developing from asymmetric trailing edge conditions. The GAW(2) airfoil which is a $13 \%$ thickness General Aviation Wing was chosen for this study because of its strong relevance in many applications. GAW(2) airfoil section was not modified in anyway to make the flow less complicated at the trailing edge. GAW(2) is an asymmetric airfoil so that the asymmetry at the trailing edge is natural, unlike the experiments referred in[8,9]. Research study [8] modified the top surface of symmetric flat plate by gluing sandpaper for the full length of the model. Study carried out in[9] used a flexible flat plate to get asymmetric conditions at the trailing edge. Research work[10] studied extensively the three-dimensional asymmetric turbulent near wake behind a swept wing with GAW(2) airfoil cross section. Our focus was concentrated in the wake region close to the trailing edge until about 75 trailing edge momentum thicknesses distance downstream of the trailing edge.

\section{Experimental Setup}

The experiments were conducted in the $300 \times 1500 \mathrm{~mm}$ boundary layer wind tunnel of the National Aerospace Laboratories, Bangalore. The GAW(2) airfoil model, having a chord length $(\mathrm{C})$ of $600 \mathrm{~mm}$ and a span of $300 \mathrm{~mm}$, was mounted vertically in the test section. The experiments were carried out at a freestream velocity of $30 \mathrm{~m} / \mathrm{s}$, providing a chord Reynold number of $1 \times 10^{6}$. GAW(2) airfoil is an asymmetric airfoil and it was not modified in any way to avoid flow complexities close to the trailing edge. Figure 1 shows the schematic of the experimental setup. Mean velocity measurements were carried out in the wake in the distance range of 1-75 trailing edge momentum thicknesses behind the trailing edge. 


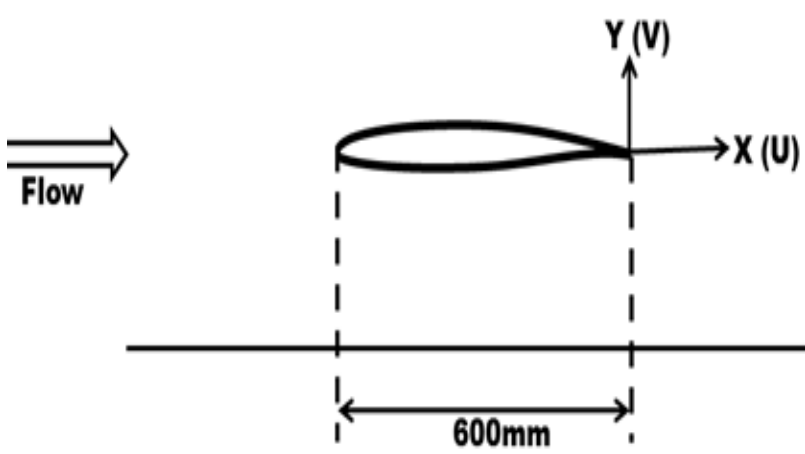

Figure 1 Schematic of the experimental setup

\section{Initial Conditions}

The development of wake behind any streamlined body depends on the initial conditions of the flow at the trailing edge. The values of displacement thickness, momentum thickness and shape factor for both top and bottom surfaces of the airfoil at a location just upstream of the trailing edge are given in the Table 1 . The values of gross parameters on the top and bottom surfaces indicate the existence of strong asymmetry at the trailing edge. Total drag was computed by measuring mean velocity profiles at three streamwise stations(namely $\mathrm{X} / \mathrm{C}=1.5,1.75$ and 2.0) and using wake survey method. The total drag coefficient at these three streamwise stations are plotted and shown in Figure 2. The plot shows very little difference in the values of total drag at these streamwise stations confirming the existence of two-dimensionality in the flow.

Table 1 Paramaters at the trailing edge

\begin{tabular}{|c|c|c|c|}
\hline Parameter $\rightarrow$ & $\delta^{*}(\mathrm{~mm})$ & $\theta(\mathrm{mm})$ & $\mathrm{H}$ \\
\hline $\begin{array}{c}\text { Top } \\
\text { surface }\end{array}$ & 3.6 & 2.1 & 1.534 \\
\hline $\begin{array}{c}\text { Bottom } \\
\text { surface }\end{array}$ & 0.8 & 0.5 & 1.416 \\
\hline
\end{tabular}

\section{Results and Discussion}

Figure 3 shows the static pressure distribution on the airfoil along the chord length. The pressure coefficient distribution indicates that the boundary layer is only under a moderate adverse streamwise pressure gradient and the flow is attached near the trailing edge. These observations are supported by the fact, as it will be seen later, that the shape factor has a value of about 1.5 at the trailing edge.

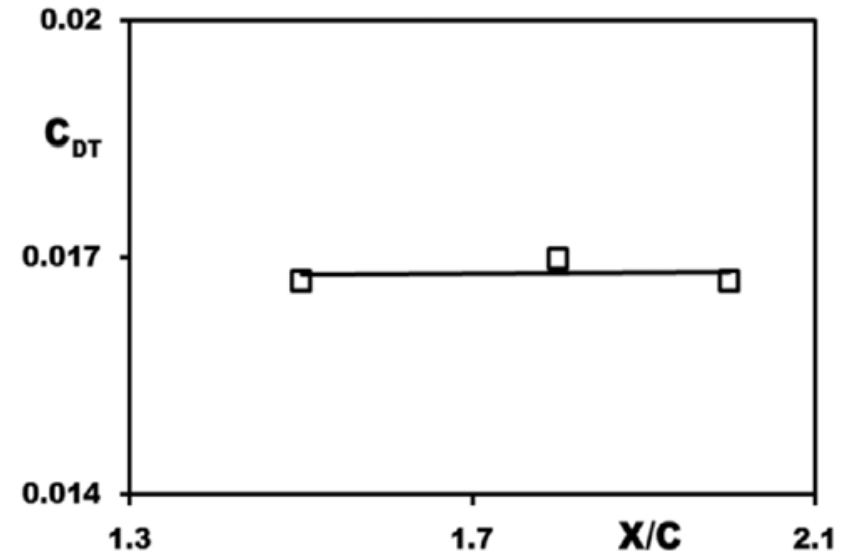

Figure 2 Total drag coefficient estimated using wake survey method at three stations.

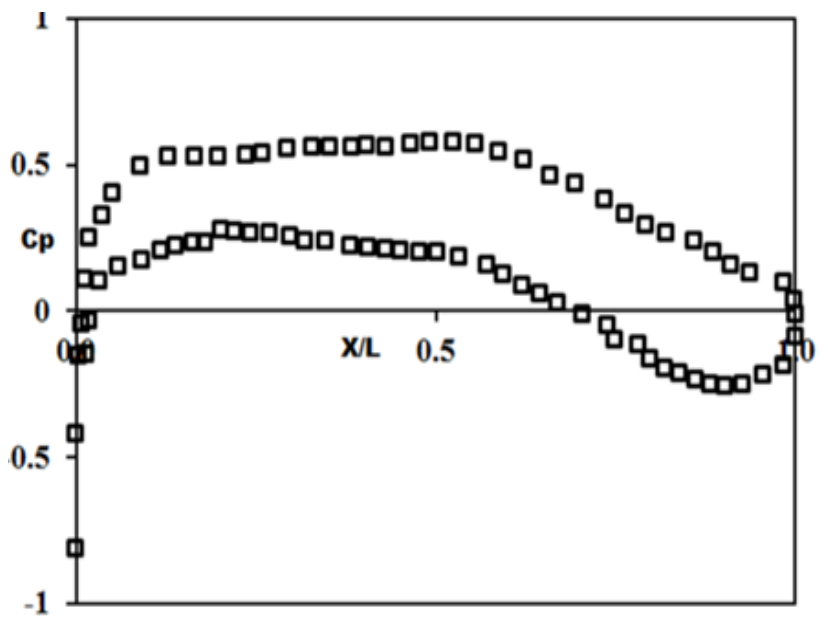

Figure 3 Pressure distribution on the airfoil model

Figure 4 shows the development of streamwise component of mean velocity profiles at various locations in the near wake. The profiles are asymmetric with respect to $\mathrm{x}$-axis and also the minimum velocity location starts shifting toward the lower side of the wake as the streamwise distance increases. Mean velocity profiles display a tendency towards symmetrisation by a distance of about 60 trailing edge momentum thicknesses. The location of $\mathrm{Y}_{\mathrm{m}}$ where the minimum velocity occurs is laterally displaced signifying that there is a net lateral (i.e. downward) transfer of momentum within the wake.

Figures 5 and 6 show the development of minimum value of mean velocity and wake half-thickness with streamwise distance in the wake. Wake half-thickness $b$ is the sum of the wake half-thicknesses on the upper and lower sides of the wake. Turbulent diffusion makes wake half-thickness and minimum velocity increase rapidly with streamwise distance.

Figures 7 and 8 show the development of momentum thickness and shape factor along the streamwise distance. One can observe a rapid decrease in the values of momentum thickness near the trailing edge and thereafter the momentum thickness is nearly constant. 


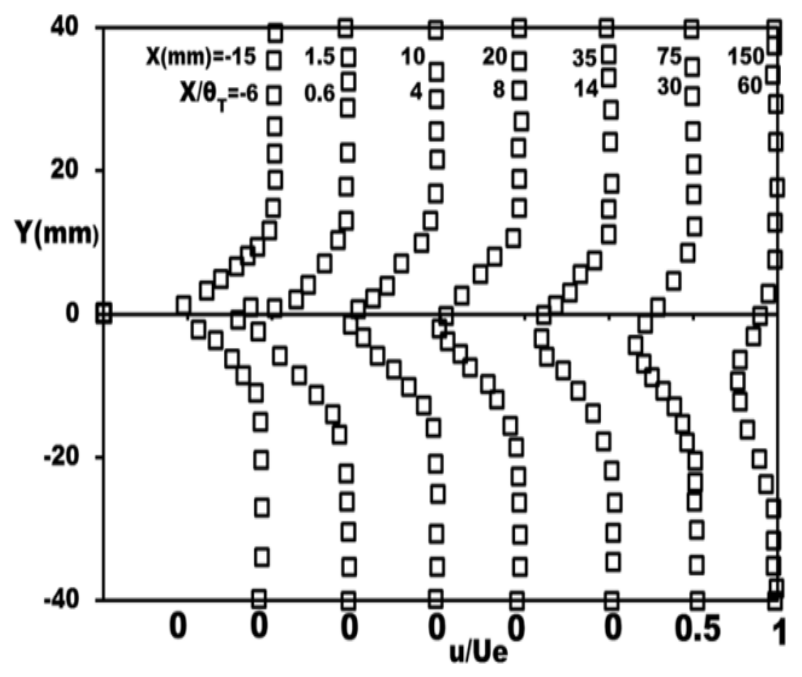

Figure 4 Profiles of streamwise component of mean velocity in the near wake

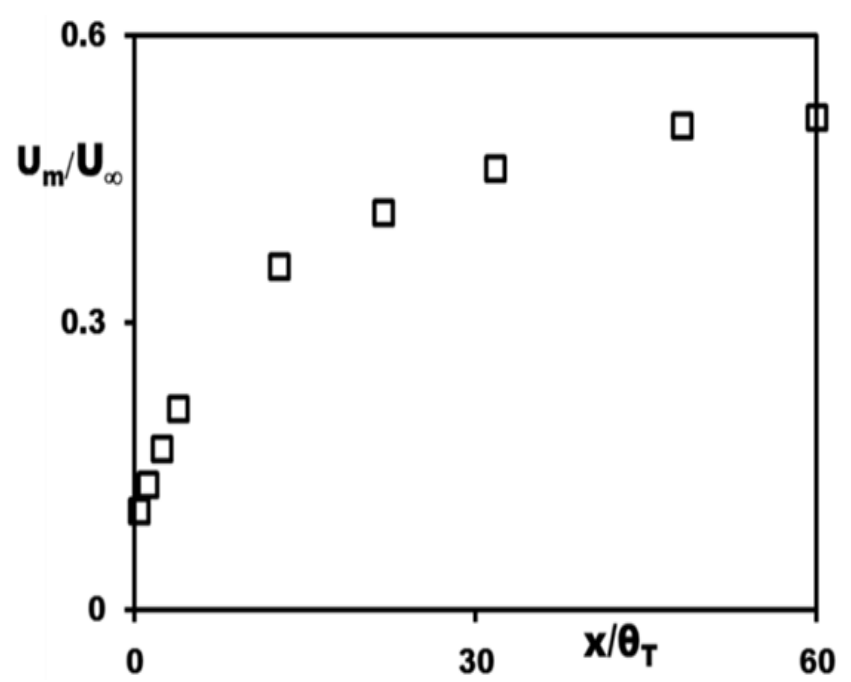

Figure 5 Variation of minimum velocity in the near wake

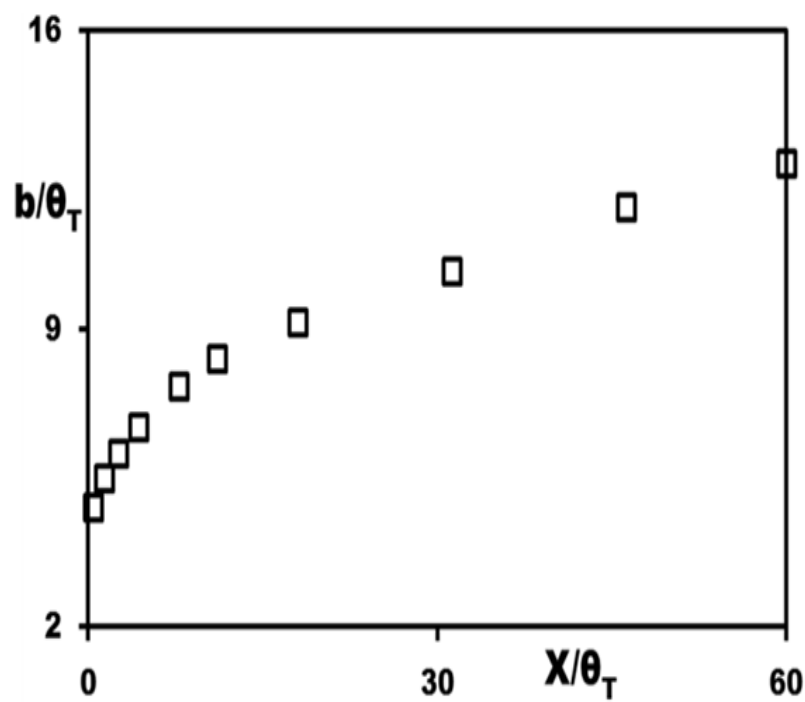

Figure 6 Variation of Wake half thickness in the near wake

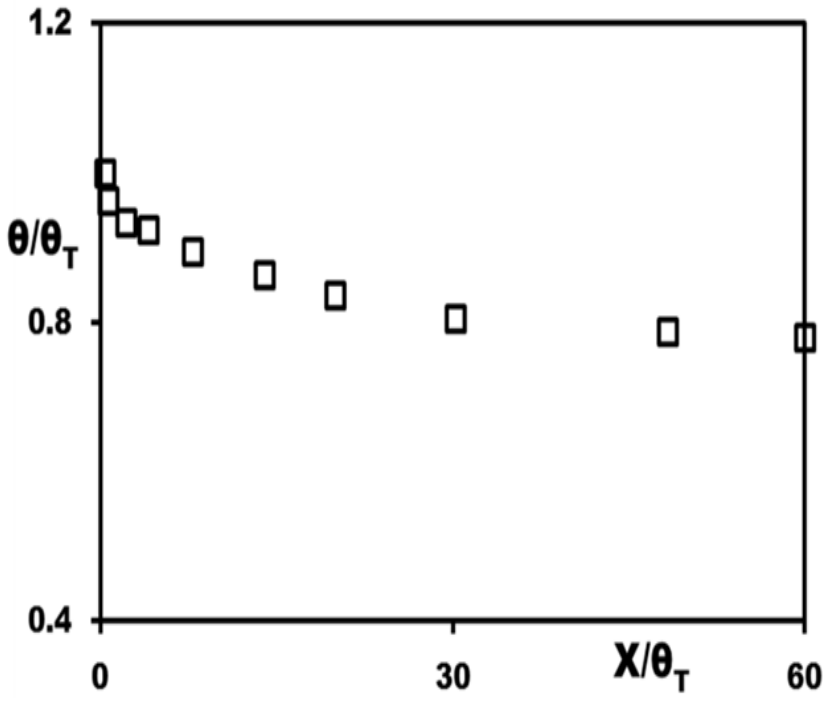

Figure 7 Momentum thickness variation in the near wake.

The shape factor evolves rapidly in the region upto $\frac{x}{\theta_{T}}=30$, and thereafter the evolution is slow; by a distance of 60 trailing edge momentum thicknesses the shape factor only slightly greater than 1, its asymptotic value at very large streamwise distances.

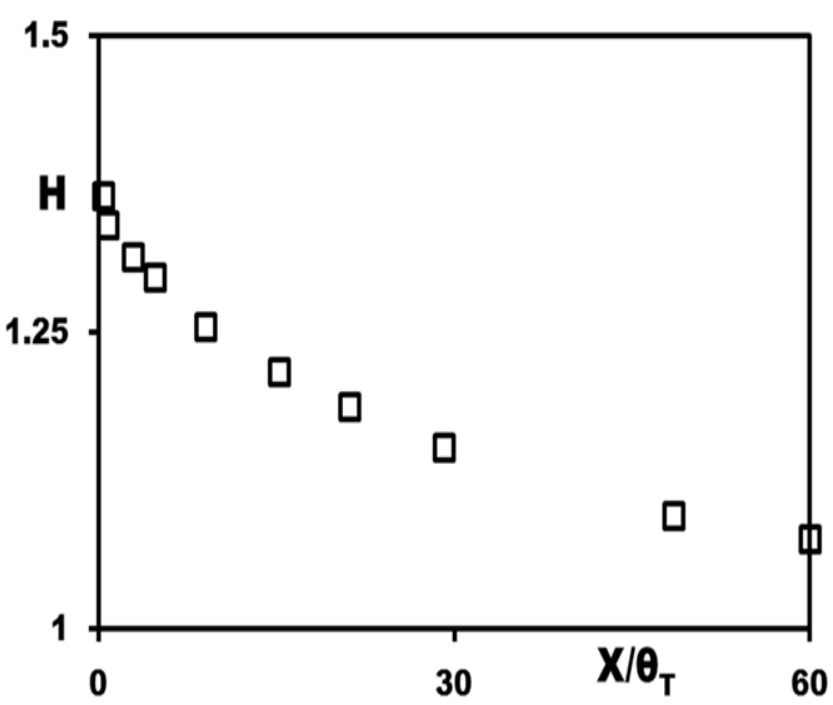

Figure 8 Variation of shape parameter in the near wake

\section{Conclusions}

Mean velocity measurements have been carried out in the near wake flow region behind a GAW(2) airfoil at low speeds. Profiles of mean velocity exhibit asymmetry close to the trailing edge and show a tendency toward symmetrisation around a distance of 60 trailing edge momentum thicknesses. The present experiments indicate that far-wake structure has not been attained within a distance of 60trailing edge momentum thicknesses. The data presented in this paper would be useful to validate 
various turbulence models, numerical schemes and computational codes.

\section{Acknowledgements}

Author acknowledges the support received from the Department of Mathematics and computational Sciences and BIUST authorities in carrying out this research

\section{References}

1. J. Andreopolous and P. Bradshaw, J. of Fluid Mech., 100, 639 (1980).

2. R. Chevary and L. S. G. Kovaznay, AIAA J., 8, 1641 (1969).

3. B. R. Ramaprian, V. C. Patel, and M. S. Sastry, AIAA J., 20, 1228 (1982).

4. N. Subaschandar and A. Prabhu, J. of Aerospace Sci. and Tech., 1, 61 (1999).

5. O. Leuchter, Turbulence in Internal Flows, Editor. S. N. B. Murthy, Hemisphere Co., Washington D.C., 371(1974).

6. B. E. Thomson, J. H. Whitelaw, J. of Fluid Mech., 157, 305 (1985).

7. M. A. Badri Narayanan, S. Raghu, and E. G. Tulapurkara, AIAA J., 23, 97 (1983).

8. B. R. Ramaprian, V. C. Patel, and M. S. Sastry, Iowa Institute of Hydraulic Research Report 231, University of Iowa, Iowa City, USA.(1981)

9. A. Nakayama, H. P. Kreplin, Phys. of Fluids, 6, 2430 (1994).

10. N. Subaschandar and A. Prabhu, J. of Aircraft, 39, 290 (2002). 